\title{
Flux-pinning properties of superconducting films with arrays of blind holes
}

\author{
S. Raedts, A. V. Silhanek, M. J. Van Bael, and V. V. Moshchalkov \\ Nanoscale Superconductivity and Magnetism Group, Laboratory for Solid State Physics and Magnetism, \\ K. U. Leuven Celestijnenlaan 200 D, B-3001 Leuven, Belgium
}

(Received 19 March 2004; published 19 July 2004)

\begin{abstract}
We performed ac susceptibility measurements to explore the vortex dynamics and the flux-pinning properties of superconducting $\mathrm{Pb}$ films with an array of microholes (antidots) and not fully perforated holes (blind holes). A lower ac shielding together with a smaller extension of the linear regime for the lattice of blind holes indicates that these centers provide a weaker pinning potential than antidots. Moreover, we found that the maximum number of flux quanta trapped by a pinning site, i.e., the saturation number $n_{s}$, is lower for the blind hole array.
\end{abstract}

DOI: 10.1103/PhysRevB.70.024509

PACS number(s): 74.78.Db, 74.25.Qt, 74.78.Na, 74.25.Ha

\section{INTRODUCTION}

The latest advances of lithographic techniques based on electron beams have allowed us to design and tailor artificial pinning centers in type II superconductors practically at will. In particular, it has been shown that periodically distributed pinning centers lead to a strong reduction of the vortex mobility and consequently to a substantial increase of the critical current when the flux line lattice is commensurate with the pinning array. ${ }^{1-7}$ So far, most of the work has been devoted to arrays of holes (antidots ${ }^{2-4}$ ) and magnetic dots. ${ }^{5-7}$ However, much less attention has been paid to the analysis of blind hole arrays. Unlike antidots, these not fully perforated holes have a thin superconducting bottom layer which allows the trapped flux to remain as separated single quantum vortices inside the pinning site. A direct confirmation of this behavior was reported by Bezryadin et al. ${ }^{8}$ who used vortex imaging by means of Bitter decoration. On top of that, a blind hole sample represents a singly connected system while an antidot sample is a multiply connected one. As has been pointed out by Moshchalkov et al..$^{2,3}$ this topological consideration might also lead to differences in the irreversible response.

In this work we perform a comparative study of the vortex dynamic response in type II superconducting $\mathrm{Pb}$ films with an array of blind holes and antidots, by ac susceptibility $\chi$ measurements. ${ }^{9,10} \mathrm{We}$ found that blind holes are less efficient pinning centers than antidots. This effect manifests itself as a lower ac shielding and consequently as a smaller extension of the linear regime. Additionally, we show that the maximum number of flux quanta, $n_{s},{ }^{11-13}$ trapped by a blind hole is systematically lower than for an antidot.

\section{EXPERIMENTAL ASPECTS}

\section{A. Sample preparation}

The used nanostructured superconducting $\mathrm{Pb}$ films were prepared as follows: first, a superconducting $\mathrm{Pb}$ layer is deposited on a $\mathrm{Si} / \mathrm{SiO}_{2}$ substrate covered by a double polymethyl metacrylate/methyl metacrylate (PMMAIMMA) resist layer in which a square lattice of square dots is predefined by electron-beam lithography (Imec vzw). The $\mathrm{Pb}$ layer is deposited in a molecular-beam epitaxy system at a working pressure of $7 \times 10^{-8}$ Torr. In order to obtain a smooth $\mathrm{Pb}$ film the substrate is cooled by liquid nitrogen $(77 \mathrm{~K})$ and the film is evaporated at a growth rate of $5 \AA / \mathrm{s}$, controlled by a quadrupole mass spectrometer. After the evaporation, the remaining resist is removed by a lift-off procedure using warm acetone. The double resist layer has an overhanging profile which avoids any contact of the deposited material on top of the resist dots with material between the dots. The final result is a $\mathrm{Pb}$ film with a square lattice of square holes. For the protection of the $\mathrm{Pb}$ samples against oxidation a 70-nm-thick Ge capping layer is finally evaporated on top of the film. In order to grow the antidot and the blind hole samples simultaneously, we first deposit a $\mathrm{Pb}$ layer (L1) on top of two identical resist dot patterns. Then, for one of them [sample $B$ in Fig. 1(b)] we carry out a lift-off procedure whereas the other [sample $A$ in Fig. 1(b)] remains unchanged. After that, a second $\mathrm{Pb}$ layer (L2) is deposited on top of both samples. Finally, the resist on sample $A$ is removed by lift-off. In this way we end up with an antidot sample (sample $A$ ) which has exactly the same thickness as the blind holes (sample $B$ ) and has been grown under identical conditions.

The data presented in this work were obtained from two sets of blind and antidot samples. Each family has a different total thickness as determined by low-angle $\mathrm{x}$-ray diffraction. In Table I we give the thicknesses of the subsequently evaporated $\mathrm{Pb}$ layers, $\mathrm{L} 1$ and $\mathrm{L} 2$, for the two studied sets of samples. Figure 1(a) shows an atomic force microscopy (AFM) image of a $5 \times 5 \mu \mathrm{m}^{2}$ surface area of the blind hole sample. The lateral size $(b=0.8 \mu \mathrm{m})$ of the holes and the period of the square array $(d=1.5 \mu \mathrm{m})$ are identical for all used samples. The periodicity of the square lattice corresponds to a first matching field of $H_{1}=\phi_{0} / d^{2}=9.2$ Oe. Here $\phi_{0}$ is the flux quantum.

\section{B. Superconducting properties}

The ac magnetization measurements were carried out in a commercial Quantum Design PPMS system with the ac field $h$ parallel to the dc field $H$ and both applied perpendicular to the sample surface. This system provides a temperature sta- 


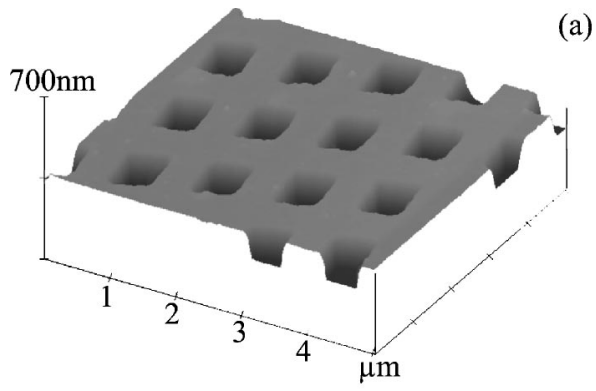

(a)

(b)
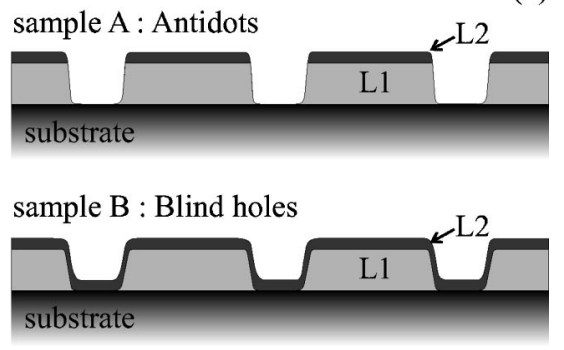

FIG. 1. (a) Atomic force micrograph (AFM) of a $5 \times 5 \mu \mathrm{m}^{2}$ area of a $\mathrm{Pb}$ film with a square array of square blind holes. (b) Schematic cross section of the patterned superconducting samples studied in this work, a blind hole sample $B$ and an antidot sample $A$. The two evaporated $\mathrm{Pb}$ layers $\mathrm{L} 1$ and $\mathrm{L} 2$ are indicated.

bility better than $0.5 \mathrm{mK}$, which is crucial for measurements near the critical temperature. The ac amplitude $h$ ranges from 2 mOe to $15 \mathrm{Oe}$ and the frequency $f$ from $10 \mathrm{~Hz}$ to $10 \mathrm{kHz}$. Since in this range of frequencies we observe that $\chi$ depends only weakly on $f$, we have chosen the same frequency $f$ $=3837 \mathrm{~Hz}$ for all measurements presented in this paper.

In order to characterize the physical properties of the different patterned films we first analyze the temperature dependence of the ac susceptibility $\chi=\chi^{\prime}+i \chi^{\prime \prime}$. The result of these measurements for set 1 of the samples is shown in the main panel of Fig. 2 at $H=5$ Oe and $h=6 \mathrm{mOe}$. The data presented in this figure have been normalized by a factor corresponding to the maximum screening, such that $\chi^{\prime}=-1$ at very low temperatures and fields. It can be seen that the $\chi^{\prime}(T)$ curve for the antidot sample $A$ (open circles) shows a very sharp superconducting transition at $T_{c 1}=7.22 \mathrm{~K}$. In contrast to that, the $\chi^{\prime}(T)$ data for blind hole sample $B$ (filled circles) first exhibits a sharp transition at $T_{c 1}$ followed by a second broader transition at $T_{c 2}=7.10 \mathrm{~K}$, below which it smoothly approaches the maximum screening. In Fig. 2 we also include the superconducting transition corresponding to a nonpatterned plain $\mathrm{Pb}$ film (triangles) with the same thickness as layer L2 and evaporated simultaneously with samples $A$ and $B$. The superconducting transition of this film coincides with the onset of the second step on sample $B$.

TABLE I. Thicknesses of Pb layers L1 and L2 for the two sets of studied samples.

\begin{tabular}{ccc}
\hline \hline & Set 1 & Set 2 \\
\hline L1 & $47.5 \mathrm{~nm}$ & $75 \mathrm{~nm}$ \\
L2 & $13.5 \mathrm{~nm}$ & $25 \mathrm{~nm}$ \\
\hline \hline
\end{tabular}

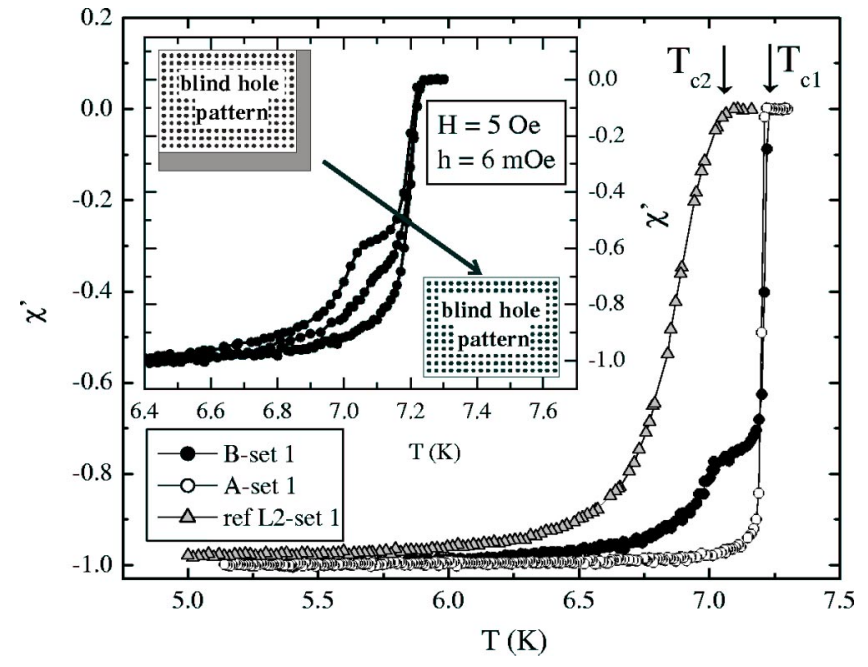

FIG. 2. Screening $\chi^{\prime}$ as function of temperature $T$ for set 1 of $\mathrm{Pb}$ films with an array of antidots ( $A$, open circles), blind holes $(B$, filled circles), and a reference plain $\mathrm{Pb}$ film with the same thickness as layer L2 (triangles), with $H=5 \mathrm{Oe}, f=3837 \mathrm{~Hz}$, and $h=6 \mathrm{mOe}$. Inset: $\chi^{\prime}$ as a function of $T$ measured on blind hole sample $B$ with the plain $\mathrm{Pb}$ contour progressively removed.

The origin of this two-step transition in the blind hole sample comes from a very narrow $\mathrm{Pb}$ border surrounding the blind hole pattern as a result of the fabrication procedure. Since the ac response is mainly given by the border of the sample, a substantial enhancement of the screening at $T_{c 2}$ is expected when this $\mathrm{Pb}$ contour turns to the superconducting state, in agreement with our observation. In order to test this, we perform $\chi^{\prime}(T)$ measurements on a similar sample while progressively removing the plain $\mathrm{Pb}$ contour, as shown in the inset of Fig. 2. Now, it can be clearly seen that the transition at $T_{c 2}$ first becomes broader and finally disappears after completely removing the plain $\mathrm{Pb}$ border. Although this undesirable contour may be eventually cut out, it helps to determine the critical temperature of $\mathrm{Pb}$ layer L2 without preparing an extra plain film. In this case, special care has to be taken in the normalization process since the total saturation value at low temperatures results from both the patterned and the unpatterned areas.

\section{RESULTS AND DISCUSSION}

Let us now compare the flux-pinning properties of the blind hole array with those obtained for the antidot array. To that end we have carried out measurements of the ac response in samples $A$ and $B$ as a function of dc field under isothermal conditions and fixed ac excitations. This is shown in the main panel of Fig. 3 for $h=0.23 \mathrm{Oe}, T=T_{c 2}=7.10 \mathrm{~K}$, and $f=3837 \mathrm{~Hz}$. In agreement with previous reports, ${ }^{2-4,14}$ the antidot sample $A$ (open symbols) exhibits clear periodic matching features at integer and rational multiples of the first matching field $H_{1}$. As we have discussed in an earlier work, ${ }^{14}$ two different regimes can be distinguished in this curve. At low fields $H<H_{3}$, a multiquanta vortex state exists and matching features appear as small steps of the screening $\chi^{\prime}$. For fields $\mathrm{H}>\mathrm{H}_{3}$ the filled pinning sites become repulsive 


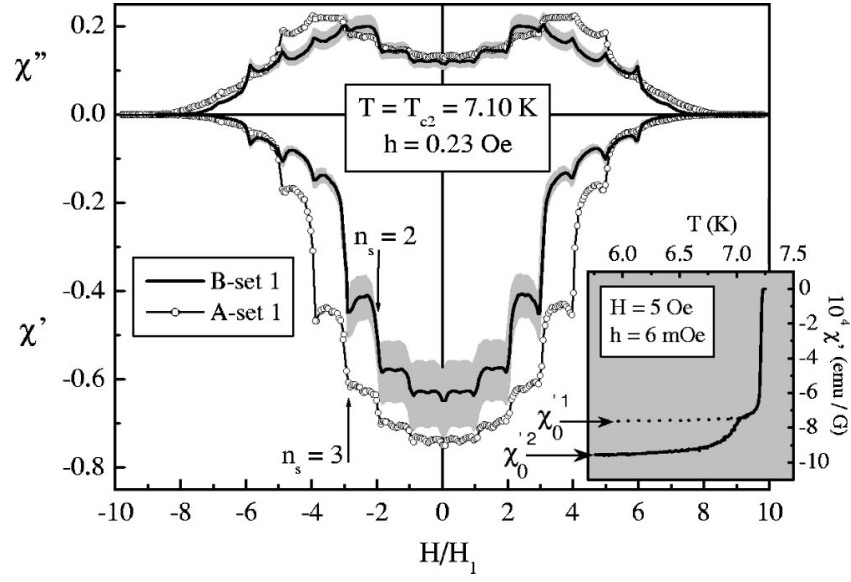

FIG. 3. Screening $\chi^{\prime}$ and dissipation $\chi^{\prime \prime}$ for films of set 1 with an array of antidots (open circles) and blind holes (thick solid line) as a function of $H / H_{1}$ for $T=T_{c 2}=7.10 \mathrm{~K}$ and $h=0.23 \mathrm{Oe}$. The inset shows the $\chi^{\prime}(T)$ transition for blind hole sample $B$, indicating the two possible saturation values used in the normalization of the signal $\chi^{\prime}$.

centers and entering vortices locate in the interstitial positions. In this regime, vortex-vortex interaction leads to highly stable vortex configurations at $H_{n}$, thus resulting in local enhancements of the screening $\chi^{\prime}(H)$. We have also shown ${ }^{14}$ that the sharp reduction in the screening at $H_{4}$ can be attributed to the higher sensitivity of the ac susceptibility in that particular range of field penetration.

As we have pointed out above, the analysis of the blind hole sample is a more subtle procedure since the signal normalization can be derived from either the saturation value corresponding to the first or the second transition. For example, data taken at $T>T_{c 2}$, where only the patterned film contributes to the signal, should be normalized using the saturation value obtained by extrapolating the first transition $\left(\chi_{0}^{\prime 1}\right)$, as shown with a dotted line in the inset of Fig. 3. A different normalization value could be obtained due to proximity effects which lead to a larger effective sample size and consequently to a higher saturation. However, no substantial change of $T_{c}$ has been detected, suggesting that the proximity effect is not relevant. In any case, the correct normalization value will lay between the two extreme values $\chi_{0}^{\prime 1}$ and $\chi_{0}^{\prime 2}$, indicated by black arrows in the inset of Fig. 3 . The result of this normalization procedure is shown as a continuous curve in the main panel of Fig. 3, whereas the extremes obtained by normalizing with $\chi_{0}^{\prime 1}$ and $\chi_{0}^{\prime 2}$ are shown as a gray painted area. The saturation value $\chi_{0}^{\prime}$ can be also estimated as $\chi_{0}^{\prime}$ $=V / 4 \pi(1-\nu)$, where $V\left(\mathrm{~cm}^{3}\right)$ is the volume of the sample and $\nu$ the demagnetization factor. ${ }^{15,16}$ For this particular sample with lateral dimensions $w_{1}$ and $w_{2}$ and thickness $\delta$, $V=w_{1} w_{2} \delta \approx 4.7 \times 10^{-7} \mathrm{~cm}^{3}$ and $1-\nu \sim\left(\delta / w_{1}\right)+\left(\delta / w_{2}\right) \sim 3.8$ $\times 10^{-5}$, so $\chi_{0} \sim 9.8 \times 10^{-4} \mathrm{emu} / \mathrm{G}$, which is very close to the experimental value $\chi_{0}^{\prime 2}=9.7 \times 10^{-4} \mathrm{emu} / \mathrm{G}$. Regardless of the chosen normalization, we can clearly see that commensurability features are also present in the blind hole sample.

A direct comparison of the $\chi(H)$ curves for samples $A$ and $B$ allows us to identify two clear differences. First, the overall screening is lower for sample $B$, indicating that blind holes provide a less efficient pinning. This effect can be intuitively understood by considering the two extreme limits of very shallow blind holes (plain film) where only intrinsic defects pin the vortices, and very deep blind holes (antidots) with a much stronger pinning force. Within this picture, it is expected that the effective pinning force grows continuously as the thickness of the bottom layer decreases. The second point to consider is that $n_{s}=2$ for blind holes, whereas $n_{s}$ $=3$ for antidots (see black arrows in the main panel of Fig. 3 ). The same difference in $n_{s}$ was found by performing dcmagnetization measurements on the same set of samples. This result is consistent with previous Bitter decoration experiments $^{8}$ showing that the difference between the saturation number of blind holes and antidots does not exceed one.

The origin of these differences can be attributed to the pinning nature of blind holes and antidots. Indeed, the interaction of a flux line with a blind hole substantially differs from the more widely investigated vortex-antidot interaction. In both cases, the normal/superconductor boundary imposes a condition to the supercurrents to flow parallel to the boundary of the hole. This effect can be modeled by introducing an image antivortex inside the hole which interacts attractively with the flux line. ${ }^{17}$ For the antidots, this attractive force acts along the total length of the flux line, whereas for blind holes we expect, as a first approximation, a smaller force proportional to the depth of the hole. This scenario becomes more complicated when considering the interaction of a flux line with an occupied blind hole. In this case, whereas flux quanta trapped by an antidot consist of supercurrents flowing around the hole, flux quanta pinned by blind holes remain as separated single-quantum flux lines with a well defined core. Now an external vortex outside of the blind hole would simultaneously feel attraction due to the image antivortex and repulsion due to the trapped vortex. Besides that, the stray field produced by vortices inside the blind holes cannot spread out freely in space since it has to be screened by the inner edges of the hole. This leads to an extra term in the interaction. For higher fillings, trapped flux lines are able to rearrange inside the blind hole, a degree of freedom absent in antidots. The repulsive interaction between these singlequantum vortices might explain the origin of the lower saturation number observed for the blind hole sample.

Let's now move on to the analysis of the ac response for temperatures above and below the critical temperature, $T_{c 2}$ of the bottom layer. For $T<T_{c 2}$, as expected, we observe the same different flux-pinning properties for blind holes and antidots, as shown in Fig. 4(a) for $T=7 \mathrm{~K}$. For $T>T_{c 2}$, an isolated plain $\mathrm{Pb}$ film with the same thickness as layer L2 is in the normal state (see Fig. 2). Although this film L2 forms the bottom layer of the blind holes, in this case it is not isolated but rather surrounded by the superconducting $\mathrm{Pb}$ bilayer which may induce superconductivity. Therefore, in this specific temperature region we expect that the pinning behavior of blind holes asymptotically approaches that of the antidots. This is indeed confirmed by the data shown in Fig. 4(b) for the same set of samples at $T=7.18 \mathrm{~K}$. The most obvious feature of this figure is the similarity between the ac response of both samples, i.e., similar ac shielding and the same saturation number. All the observations reported for set 

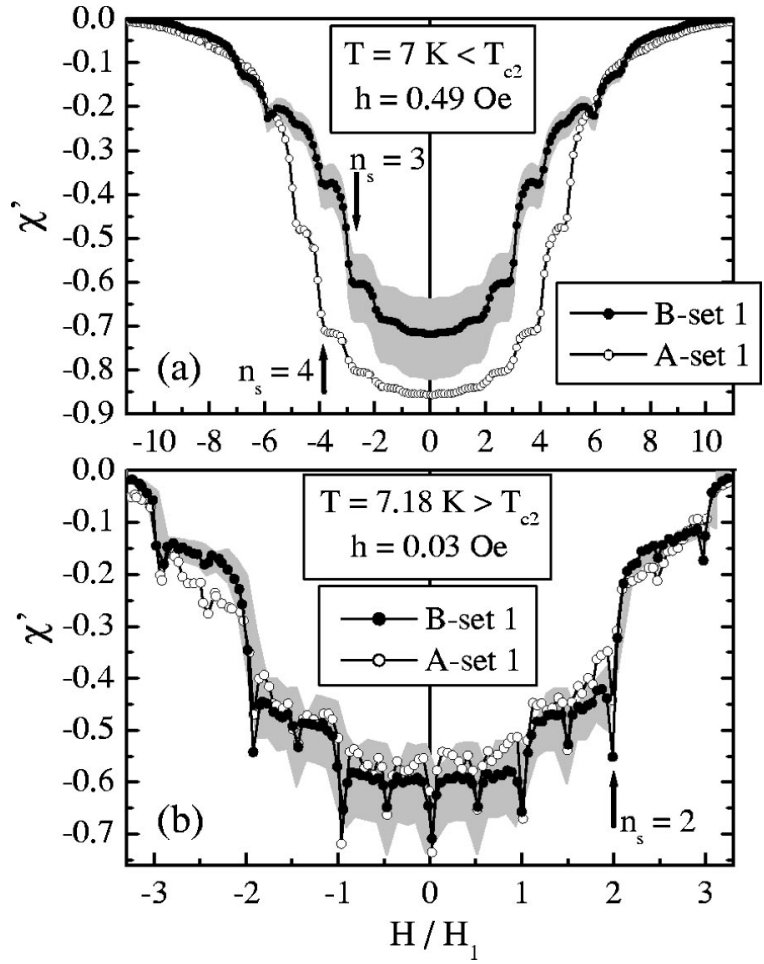

FIG. 4. Screening $\chi^{\prime}$ as function of $H / H_{1}$ for $\mathrm{Pb}$ films of set 1 with an array of blind holes (filled symbols) and antidots (open symbols) with (a) $T=7 \mathrm{~K}<T_{c 2}$ and $h=0.49 \mathrm{Oe}$ and (b) $T$ $=7.18 \mathrm{~K}>T_{c 2}$ and $h=0.03 \mathrm{Oe}$.

1 of the samples were also reproduced for set 2 of the samples. These results are shown in Fig. 5. In this case, samples $A$ and $B$ have the same $T_{c}=7.22 \mathrm{~K}$, as shown in the inset of Fig. 5.

An alternative way to investigate the pinning properties of blind holes and antidots is to analyze the different ac vortex dynamic regimes. ${ }^{18,19}$ For very low ac drives, all vortices

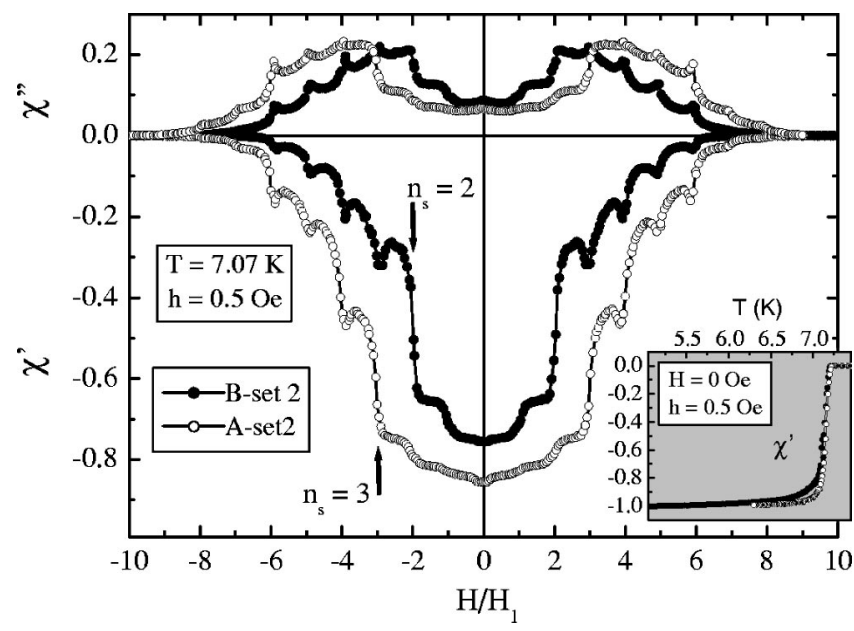

FIG. 5. Screening $\chi^{\prime}$ and dissipation $\chi^{\prime \prime}$ as a function of $H / H_{1}$, for $\mathrm{Pb}$ films of set 2 with an array of antidots (open circles) and blind holes (filled circles) for $T=7.07 \mathrm{~K}, f=3837 \mathrm{~Hz}$, and $h$ $=0.5$ Oe. The inset shows the temperature dependence of the normalized screening $\chi^{\prime}$ for the samples $A$ and $B$.
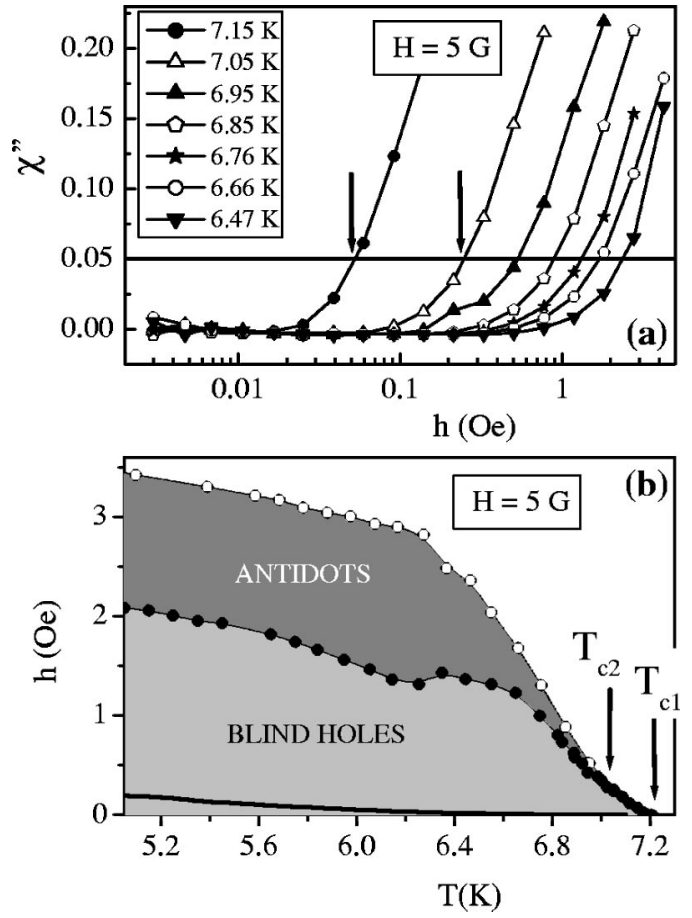

FIG. 6. (a) Dissipation $\chi^{\prime \prime}$ as function of the ac field $h$ for an array of antidots at several $T, f=3837 \mathrm{~Hz}$, and $H=5$ Oe. Arrows indicate the onset of the nonlinear response according to the chosen criterium $\chi^{\prime \prime}=0.05$ (horizontal line). (b) Phase boundary of the linear regime for samples $A$ and $B$ of set 1 , for $H=5$ Oe and $f$ $=3837 \mathrm{~Hz}$. This boundary is obtained using a dissipation criterium $\chi^{\prime \prime}=0.05$, as shown in (a) for antidot sample $A$. The continuous line indicates the boundary of the linear regime for a reference nonpatterned $\mathrm{Pb}$ film with the same thickness as layer $\mathrm{L} 2$.

oscillate inside the corresponding individual pinning potentials. This so-called linear regime is characterized by an $h$-independent screening together with a very low dissipation. ${ }^{20,21}$ As the ac drive is increased, vortices eventually overcome the pinning well switching to a more dissipative regime with an $h$-dependent screening. The boundary between these two regimes is mainly determined by the strength of the pinning centers. Consequently, the stronger the pinning, the larger the extension of the linear regime. Experimentally, a reliable criterium to determine the onset of nonlinearity is given by a dissipation $\chi^{\prime \prime}(h)=0.05$, as shown in Fig. 6(a) for sample $A$ of set 1 at several temperatures. Performing this procedure for samples $A$ and $B$, we can compare the dynamic diagrams $h(T)$ of antidot and blind hole samples [see Fig. 6(b)]. Most obvious in Fig. 6(b) is the smaller extension of the linear regime for the blind hole sample $B$. This is a clear indication that the blind hole array produces a weaker pinning potential, in agreement with our previous observations. In addition, for temperatures $T>T_{c 2}$, the two boundaries collapse on a single line. This result is consistent with the fact that for $T>T_{c 2}$ the thin layer at the bottom of the blind holes approaches a normal metal, thus turning to the behavior of the antidot sample. Figure 6(b) also includes the dynamic diagram $h(T)$ for a reference film with the same thickness as layer L2. As expected, the very low effective pinning of the plain film results in a substantial 
smaller extension of the linear regime in comparison with the patterned samples $A$ and $B$.

It is important to stress that there is also a difference in the depinning process of vortices trapped by antidots and blind holes. On one hand, single-quantum vortices trapped by the blind holes are able to depin one by one. On the other hand, as has been pointed out by Priour and Fertig, ${ }^{22}$ in the case of multiquanta vortices (without rigid core) trapped by antidots, the driving current elongates the vortex core which can eventually reach the neighbor pinning site, thus allowing the vortex to hop from site to site. All these considerations should be taken into account in order to theoretically analyze the pinning properties of blind holes.

\section{CONCLUSION}

We have used ac susceptibility to perform a comparative study of the flux-pinning properties of an array of antidots and blind holes. We show that antidots are more efficient pinning centers than blind holes where the superconducting film is not fully perforated. Consequently, a reduced screening for the blind hole system is observed. Therefore, the strength of the pinning potential can be gradually tuned by varying the depth of blind holes. On top of that, the saturation number $n_{s}$, defined as the maximum number of flux quanta that a pinning site can hold, is higher for antidots than for blind holes, in agreement with previous reports. The linear regime, in which vortices oscillate inside the pinning potential, has a smaller extension for the blind hole sample, indicating that blind holes provide a weaker pinning potential. Finally, we discussed the ac response for temperatures above the critical temperature of the bottom layer and found that the pinning behavior of blind holes approaches the behavior of antidots.

\section{ACKNOWLEDGMENTS}

This work was supported by the Fund for Scientific Research-Flanders (FWO-Vlaanderen), the Belgian InterUniversity Attraction Poles (IUAP), the Research Fund K.U.Leuven (GOA), and by the European ESF VORTEX programs. M.J.V.B. received additional support from FWO. We thank J. Van de Vondel and C. de Souza Silva for helpful discussions.
${ }^{1}$ O. Daldini, P. Martinoli, J. L. Olson, and G. Berner, Phys. Rev. Lett. 32, 218 (1974).

${ }^{2}$ V. V. Moshchalkov, M. Baert, V. V. Metlushko, E. Rosseel, M. J. Van Bael, K. Temst, R. Jonckheere, and Y. Bruynseraede, Phys. Rev. B 54, 7385 (1996).

${ }^{3}$ V. V. Moshchalkov, M. Baert, V. V. Metlushko, E. Rosseel, M. J. Van Bael, K. Temst, and Y. Bruynseraede, Phys. Rev. B 57, 3615 (1998).

${ }^{4}$ M. Baert, V. V. Metlushko, R. Jonckheere, V. V. Moshchalkov, and Y. Bruynseraede, Phys. Rev. Lett. 74, 3269 (1995).

${ }^{5}$ M. J. Van Bael, J. Bekaert, K. Temst, L. Van Look, V. V. Moshchalkov, Y. Bruynseraede, G. D. Howells, A. N. Grigorenko, S. J. Bending, and G. Borghs, Phys. Rev. Lett. 86, 155 (2001).

${ }^{6}$ M. J. Van Bael, K. Temst, V. V. Moshchalkov, and Y. Bruynseraede, Phys. Rev. B 59, 14674 (1999).

${ }^{7}$ J. I. Martín, M. Vélez, J. Nogués, and I. K. Schuller, Phys. Rev. Lett. 79, 1929 (1997).

${ }^{8}$ A. Bezryadin, Y. N. Ovchinnikov, and B. Pannetier, Phys. Rev. B 53, 8553 (1996).

${ }^{9}$ V. V. Metlushko, U. Welp, G. W. Crabtree, Z. Zhang, S. R. J. Brueck, B. Watkins, L. E. DeLong, B. Ilic, K. Chung, and P. J. Hesketh Phys. Rev. B 59, 603 (1999).

${ }^{10}$ V. V. Metlushko, L. E. DeLong, M. Baert, E. Rosseel, M. J. Van Bael, K. Temst, V. V. Moshchalkov, and Y. Bruynseraede, Eu- rophys. Lett. 41, 333 (1998).

${ }^{11}$ G. S. Mkrtchyan and V. V. Schmidt, Zh. Eksp. Teor. Fiz. 61, 367 (1971) [Sov. Phys. JETP 34, 195 (1972)].

${ }^{12}$ M. M. Doria and G. Figueira Zebende, Phys. Rev. B 66, 064519 (2002).

${ }^{13}$ H. Nordborg and V. M. Vinokur, Phys. Rev. B 62, 12408 (2000).

${ }^{14}$ A. V. Silhanek, S. Raedts, M. J. Van Bael, and V. V. Moshchalkov, Phys. Rev. B (to be published 1 August 2004).

${ }^{15}$ J. R. Clem and A. Sanchez, Phys. Rev. B 50, 9355 (1994).

${ }^{16}$ C. P. Poole, H. A. Farach, and R. J. Creswick, Superconductivity (Academic Press, San Diego, 1995).

${ }^{17}$ A. Buzdin and D. Feinberg, Physica C 256, 303 (1996).

${ }^{18}$ G. Pasquini, L. Civale, H. Lanza, and G. Nieva, Phys. Rev. B 59, 9627 (1999); L. Civale, A. V. Silhanek, and G. Pasquini, in Studies of High Temperature Superconductors, edited by Anant Narlikar (Nova Science, New York, 2003), Vol. 47.

${ }^{19}$ A. V. Silhanek, S Raedts, M. J. Van Bael, and V. V. Moshchalkov, Eur. Phys. J. B 37, 19 (2004).

${ }^{20}$ G. Pasquini, P. Levy, L. Civale, G. Nieva, and H. Lanza, Physica C 274, 165 (1997).

${ }^{21}$ A. V. Silhanek, S. Raedts, M. Lange, and V. V. Moshchalkov, Phys. Rev. B 67, 064502 (2003).

${ }^{22}$ D. J. Priour and H. A. Fertig, Phys. Rev. B 67, 054504 (2003). 